http://jmscr.igmpublication.org/home/ ISSN (e)-2347-176x ISSN (p) 2455-0450 crossref DOI: https://dx.doi.org/10.18535/jmscr/v7i7.134

\title{
Reduction of Inappropriate Use of Oxygen by Standardizing Initiation of Simple Check List among Neonates Admitted in Newborn Care Unit: A Quality Improvement Initiative
}

\author{
Authors \\ Narendra Behera ${ }^{1}$, Dr Jayanti Prava Behera ${ }^{2}$ \\ ${ }^{1}$ Associate Prof. Pediatric, S.C.B Medical College, Cuttack, India \\ ${ }^{2}$ Prof. Pharmacology; B.B Medical College, Bolangir, India
}

\begin{abstract}
Introduction: Preterm and term infants are more frequently exposed to high concentrations of oxygen for prolonged periods. When supplemental oxygen is needed for care, it will be prudent to avoid fluctuations in SpO2. The definition of the safest level of oxygen saturations in the neonate remains an area of active research. On the basis of the recently published evidences, the most suitable approach would be to set an alarm limits between 90 and $95 \%$.

Objective: To reduce the inappropriate use of $\mathrm{O} 2$ (concentration and duration) by implementation of standard protocol, which is done by quality improvement strategy.

Materials and Methods: This is a quality improvement study conducted in a tertiary care children's health institution in India. Pre interventional and post interventional data are collected from hospital records of 69 and101 hospitalized newborn cases respectively. By Q.I method, first prioritization of problem and research team formation, then clear aim statement about study is discussed prior to data collection. In $2^{\text {nd }}$ step analysis of problems and the team members tried to solve this problem by using the fish bone analysis method. Then the changes are made as per PDSA cycle (CME about implementation of new protocol, reorientation of staffs, some changes made in emergency department. Initially smaller changes then multiple and larger events done). The final data are analyzed and compare with baseline data.

Results: There reduction of duration and concentration of $O 2$ use is observed as $<2$ days duration in final data i.e $69.7 \%$ which is significantly more than base line data (23\%). Appropriate use of O2 among neonates is significantly increased after implementation of quality improvement strategy (67.96\%) over base line data (19\%). In final data mean 02 therapy is 1-2 days in comparison with base data i.e 3-5 days. There are no significant changes in outcome parameters.
\end{abstract}

Conclusion: Practice recommendation of $\mathrm{O} 2$ saturation targets newborn cared with minimizing oxygen therapy and toxicity.

Keywords: Oxygen, newborn, SPO2, preterm, free radicals.

\section{Introduction}

Oxygen is very essential in newborn health care because many conditions that affect babies in the first days of life which can result in low levels of oxygen in the body. Hypoxemia is a life-threatening condition that results in increased mortality and morbidity among neonates. Prematurity and respiratory distress syndrome (surfactant deficiency), pneumonia and other severe infections, asphyxia and difficulties in the transition from foetal to neonatal life can all result in hypoxemia. Supplemental oxygen is an essential lifesaving treatment during this period ${ }^{[1]}$. The therapeutic use of oxygen in neonatal period is considered as double edged sword because of its beneficial and toxic effects. During the $1^{\text {st }}$ month of life, hyperoxia, inflammation, episode of hypoxia-reoxygenation 
and free iron are appear to be the sources of increased ROS release which may cause tissue injury. Oxidative stress in neonates is mainly due to decreased antioxidants and increased reactive oxygen species (ROS), or both. Studies reported that antioxidant capacity, in preterm newborns is lesser than term babies. The mechanisms of ROS generation are: mitochondrial respiratory chain, free iron and Fenton reaction, inflammation, hypoxia and/or ischemia, reperfusion, and hyperoxia ${ }^{[2]}$. Oxidative stress due hyperoxia has been recognized to be responsible for generalized tissue damage and in particular causes injury to lung, CNS, retina, red blood cells. When supplemental oxygen is needed for new born care, it should be prudent to avoid changes and fluctuations in $\mathrm{SpO}_{2}$. The definition of the safest level of oxygen saturations in the neonate remains an area of active research ${ }^{[3]}$. Current WHO recommendations and clinical guidelines address several aspects of oxygen therapy. Clinical indications for oxygen use include resuscitation of preterm infants and advanced resuscitation of term infants as well as the full spectrum of respiratory illness from mild hypoxemia to moderate/severe respiratory distress and respiratory failure ${ }^{[4]}$.

Currently, on the basis of the published evidences, the most suitable approach would be to set alarm limits between 90 and $95 \%$. It should allow to avoid $\mathrm{SpO}_{2}$ values associated with potential hypoxia and/or hyperoxia as the risk of tissue damage due to oxidative stress in perinatal period should not be underestimated $^{[5]}$. Other study in New Zealand reported that there was heterogeneity for mortality between the original oxygen saturation algorithm and the revised algorithm which resulted in clearer separation of the groups. The peak median saturation for infants receiving supplementary oxygen is $89 \%$ vs $92 \%{ }^{[6] \text {. }}$

In most of the health facilities in India, WHO guide line is not followed and over dose of $\mathrm{O} 2$ used irrationally which lead to several disease conditions leading to increased morbidity and mortality. At the same time the available resources are used unnecessarily without indication and create a scarcity during emergency requirement. So there is a definite gap between requirement and utilization which lead to economic loss with the cost of precious lives of neonates of our country ${ }^{[7]}$.

On this back ground this study was conducted to evaluate the effectiveness of reduction of inappropriate use of Oxygen by standardizing initiation of simple check list among neonates admitted in newborn care unit by a quality improvement study.

\section{Aim}

To reduce irrational use of oxygen in new born unit among sick neonates by using quality improvement method.

\section{Objectives}

- Assessment the need of o2 therapy as per o2 requirement protocol (pulse-oxymetry)

- Assessment of duration and amount of $\mathrm{O} 2$ therapy in sick neonates in practice.

- Comparison of base line data and final data of various parameters and outcome before implementation of new QI strategy.

\section{Materials and Methods}

This was a QI (quality improvement) study conducted in the newborn unit (casualty, newborn word, ACRC (advance care resource centre) and SNCU (special newborn care unit) of pediatrics IPD of a tertiary health care center in India from $1^{\text {st }}$ June to $31^{\text {st }}$ July, 2018. This study protocol was approved by IEC of this institution. This study was conducted as per POCQI (point of care quality improvement) model 1. Identify problem, forming team and write an aim statement. 2. Analyze the problem and majoring quality of care. 3. Developing changes and testing it.4.Sustaining improvement. This project was planned in 3 phases, such as 1 . Baseline Data collection (preintervention) phase for 7 days, 2 . Implementation (orientation of changes) phases for 1 month and 3. Final data collection phase for 7 days. For this study a QI team is constituted by including 1 associate professor( team leader), 2 assistant professors, 4 post graduate students, 3 nursing staffs in charge of different sections of NBU. Graveness 
of the study protocol discussed amongst the team members. A CME conducted in the department on QI project as per POCQI model. Awareness on issues on $\mathrm{O} 2$ therapy discussed and guide lines on o2 recommendation are discussed. Base line data collection and analysis of data (\% uses of $\mathrm{O} 2$, risk analysis, $\%$ of decreases 02 uses and outcome majors and plotted in flow chart. Then majors taken to decrease the uses of $\mathrm{o} 2$, awareness related to 02 therapy and toxicity, work place up gradation as per fish bone analysis and changes made as per PDSA(plan, do, study, act) cycle. This new plans and changes made are reflected in emergency. Comparison of final data with base line data done then multiple changes in the PDSA cycle done as per pros and cons. Negative results abounded and positive results are accepted. A total of 69 cases in pre intervention phase and 101 cases in intervention phase are included in the study. New born cases 0 1month of age, all the sexes, receiving oxygen therapy are selected.

\section{O2 Therapy Recommendation}

Newborn with hypoxemia closely monitored by pulse-oxymetry $(<90 \%)$. O2 therapy will be discontinued in a chronically stable newborn. Stop O2 therapy when $\mathrm{SPO} 2>90 \%, \mathrm{O} 2$ to be discontinued for 15 minute then newborn look for clinical signs of hypoxemia and spo2 level. Target O2 saturation done by using alarm limit. Observation of $\mathrm{O} 2$ saturation for 2-3 seconds and make $\mathrm{O} 2$ adjustment for stable saturation level for 3-5 minutes. It also observed if target not reaching at appropriate level give extra $\mathrm{O} 2$ supply. If SPO2 level stable for 30 minute stop $\mathrm{O} 2$ supply. An attempt for weaning then decrease nasal flow of $\mathrm{O} 2$ to 0.5 litre/minute, then discontinue the $\mathrm{O} 2$ supply ${ }^{[8],[9]}$.

\section{Study Procedure}

At beginning of the study there is a discussion among senior faculties with team leader based on their experience in their facility to prioritizing the problem on 4 important points such as important to patient outcome, affordable in terms of time and resources and easy to measure ${ }^{[10]}$.
Data collection procedure: Data are collected in a predesigned format.

The base line data regarding various aspect of oxygen therapy like whether recommended as per oxygen guide lines protocol (emergency signs, priority sign and less specific sign), duration of o2 therapy and outcome were collected ${ }^{[4]}$. Emergency signs if any like obstructed or absent breathing, severe respiratory distress,cyanosis,signs of heart failure,signs of shock, severe sepsis, heart rate with weak pulse $(>=160 / \mathrm{m} \&<=100 / \mathrm{min})$, low or unmeasurable BP (systolic $<40 \mathrm{mmhg}$ ), coma (very low level of consciousness $\mathrm{GCS}<=8$ ), convulsion, signs of severe dehydration $\mathrm{H} / \mathrm{O}$ diarrhea(any two signs) are noted. Priority signs if any are also recorded such as preterm, temp hypothermia and or hyperthermia,trauma or other urgent condition, pallor (Severe), respiratory distress, signs of hypoglycemia, $\mathrm{SPO}_{2}<90 \%$, central cyanosis, nasal flaring, inability to drink or feed(Respiratory distress), grunting with every breath,depressed mental status -drowsy, lethargy etc. Less specific signs if any are also recorded such as severe lower chest in drawing, respiration rate $>=70 / \mathrm{m}$, head nodding synchronous with respiration ,notable birth asphyxia (APGAR Score<=3), TTN,HMD,MAS, hypoglycemia,sick prematurity, apnea of primary bradycardia <100/min, apnea pathological and hypoventilation, birth weight $<1.5 \mathrm{~kg}$, gestational age $<32$ weeks etc.

The outcome data in terms of discharged, death, LAMA and referred are recorded. After fish bone analysis of baseline data the QI team discussed about existing practice of $\mathrm{O} 2$ use and possible barriers in implementing the suggested strategy. Then how to implement the new strategy to improve the quality care is finalized. After the base line phase the training of the team members is conducted. PDSA cycle is done involving team members on 5 points like, duration of oxygen therapy to be reduced from the existing practice, given as per the specific indication, with signs of emergency, with minimum investigation prior to therapy etc, persons involved $\mathrm{n}$ follow up, place, duration of study and expected outcome. Training material is prepared by 
the QI team. The written material is widely circulated among other doctors and nurses for implementation. Posters regarding new protocol were displayed at several selective places to aware doctors and nursing staffs about the new strategy regarding oxygen use.

Finally, following changes are made in the system: (1) training of doctors and neonatal nurses, (2) finalizing the new protocol (3) supervised by QI team members (4) displaying the guidelines for $\mathrm{O} 2$ use at different places of NBU (5) Assigning the team members to collect data regarding various aspect $\mathrm{O} 2$ use in a predesigned case record form prepared by team members.

Post-intervention phase dealt with follow up of patients daily during the hospital stay QI team meets every week to collect feedback from all stakeholders and review each important aspects of feedback and decide to bring any changes in the system, if necessary.

\section{Statistical Analysis}

Both base line data and final data are compiled and analyzed by using statistical soft ware, Graph pad prism version.5. The categorical data are analyzed by descriptive analysis and expressed in percentages. The comparison between base line data and final data are done by Chi-square test.

\section{Results}

Fig No.1 Distribution of O2 Therapy among Neonates

\section{Data 1}

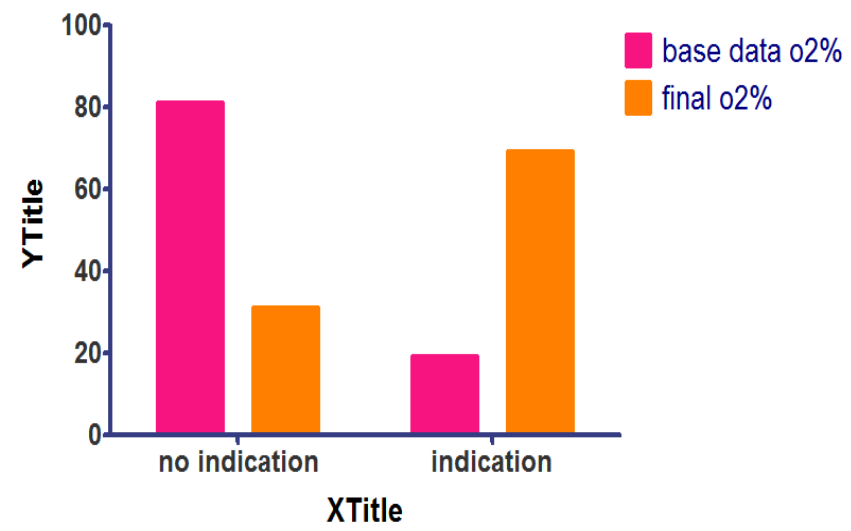

$\mathrm{N}=69$ (base line data), $\mathrm{n}=101$ (final data), Data expressed in $\%$ and analysed by Chi square test, $\mathrm{x}^{2}$ 4.448 , df- $1, \mathrm{p}<0.05$.
More percentage $(81 \%)$ of cases were received $\mathrm{O} 2$ therapy without indication in base line data whereas significantly less percentage $(32.03 \%)$ of cases received $\mathrm{O} 2$ therapy in final data. There was significant rise of $\mathrm{O} 2$ uses $(67.69 \%)$ in final data as per indication.

Fig No.2 Oxygen Used as per Emergency Signs

Data 1

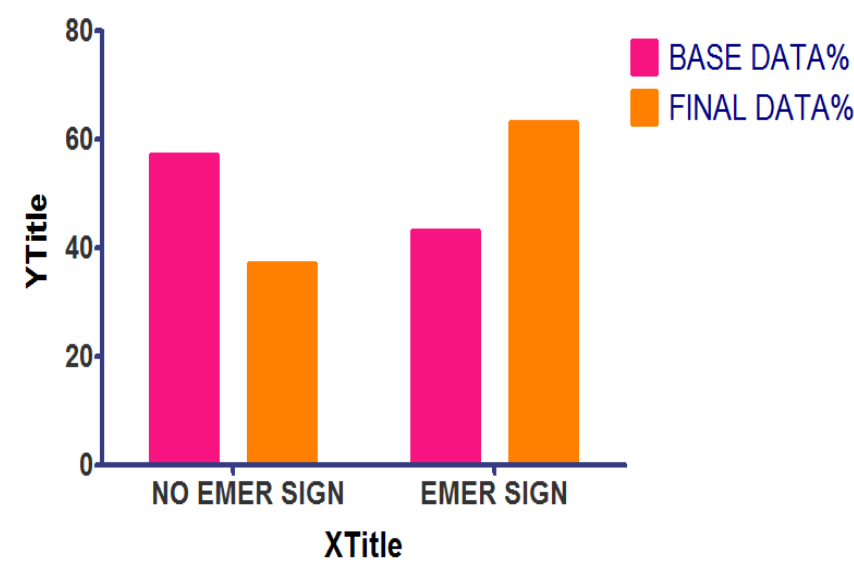

$\mathrm{N}=69$ (base line data), $\mathrm{n}=101$ (final data), Data expressed in $\%$ and analysed by Chi square test, $\mathrm{x}^{2}$ $8,029, \mathrm{df}-1, \mathrm{p}<0.001$.

Fig No.2 showed that there was significantly more percentage of cases received $(62.5 \%) \mathrm{O} 2$ therapy as per emergency sign in final data compared with that of baseline data (43\%). In base line data more percentage of cases received $\mathrm{O} 2$ therapy without any emergency sign (57\%).

Fig No. 3 Use of Oxygen as per Specific Sign

Data 1

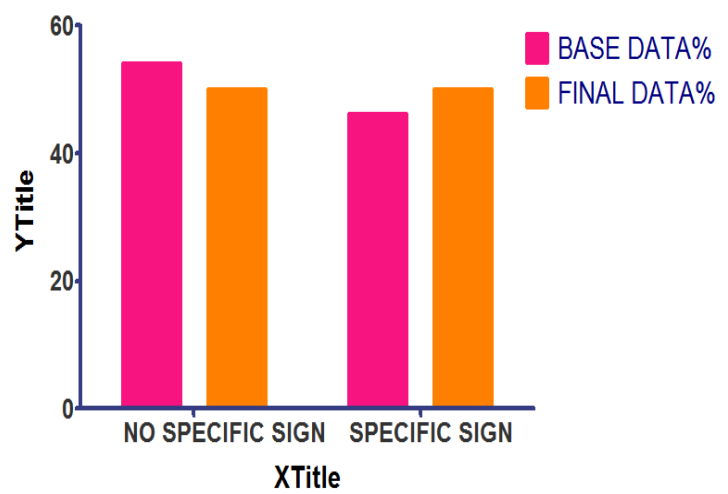

$\mathrm{N}=69$ (base line data), $\mathrm{n}=101$ (final data), Data expressed in $\%$ and analysed by Chi square test, $\mathrm{x}^{2}$ 0.3205 ,df- $1, \mathrm{p}>0.05$

There was no significant difference between the two groups among $\mathrm{O} 2$ used as per specific sign. Though 
there was slightly higher percentage $(54 \%)$ of cases received $\mathrm{O} 2$ therapy as per specific indication in final data compared with baseline data.

Fig. No.4 Oxygen as per Priority Sign

\section{Data 1}

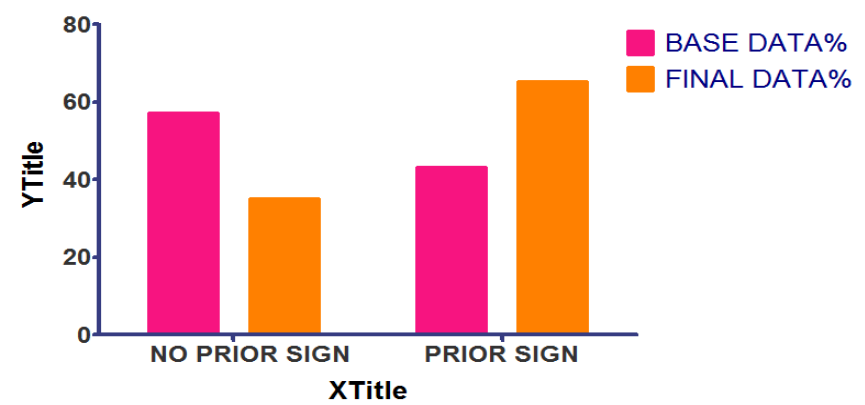

$\mathrm{N}=69$ (base line data), $\mathrm{n}=101$ (final data), Data expressed in $\%$ and analysed by Chi square test, $\mathrm{x}^{2}$ 8,029, df- $1, \mathrm{p}<0.001$

Fig No.4 showed that in final data after intervention the $\mathrm{O} 2$ used as per priority sign was occurred in significantly more $(64.84 \%)$ of cases than that of baseline data(43\%) whereas maximum percentage of cases used not as per priority sign (57\%) in baseline data.

Fig No.5 O2 Used After Minimum Investigation

Data 1

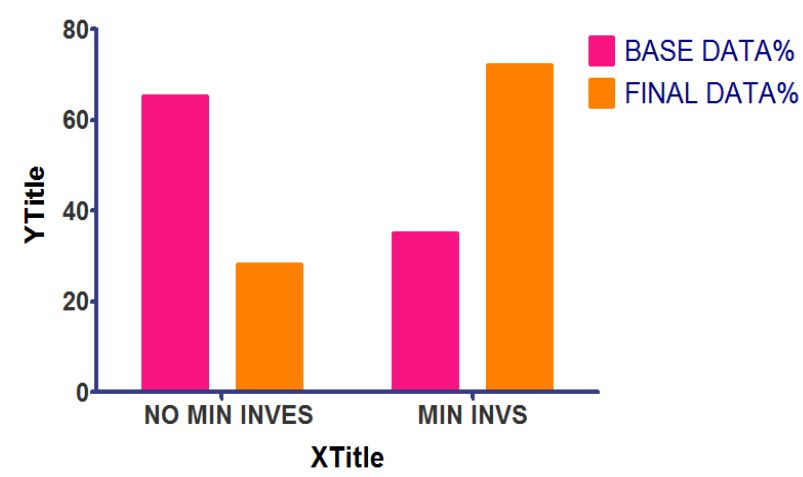

$\mathrm{N}=69$ (base line data), $\mathrm{n}=101$ (final data), Data expressed in $\%$ and analysed by Chi square test, $\mathrm{x}^{2}$ 27.51,df-1, p <0.0001

In Fig.No.5, it was clearly observed that maximum percentage of cases received $\mathrm{O} 2$ therapy with minimum investigation in final data group $(71.87 \%)$ which was significantly less in basal data group $(35 \%)$.
Fig No.6 Duration of O2 Used

Data 1

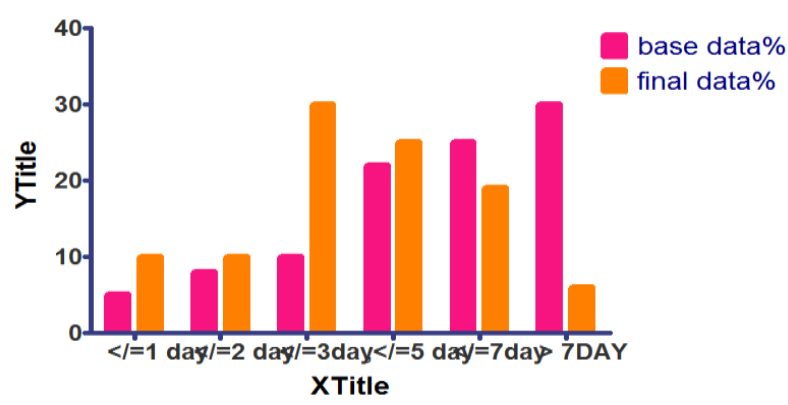

$\mathrm{N}=69$ (base line data), $\mathrm{n}=101$ (final data), Data expressed in $\%$ and analysed by Chi square test, $\mathrm{x}^{2}$ 28.90,df-5, $\mathrm{p}<0.001$

Duration wis $\mathrm{O} 2$ uses maximum around day 7 in base data where as maximum use of $\mathrm{O} 2$ limited to day3.

Fig No.7 O2 Used Duration: <2 Days Vs >2 Days

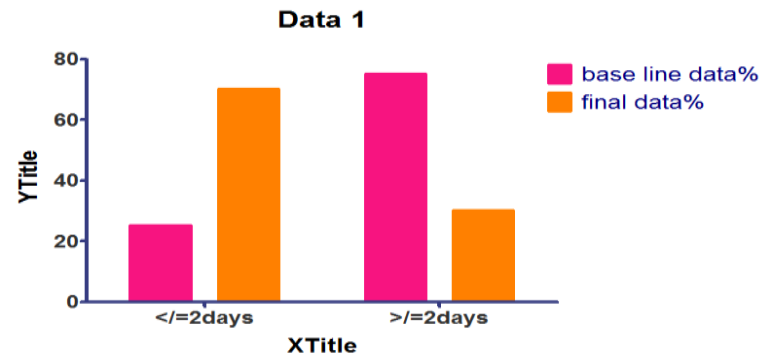

$\mathrm{N}=69$ (base line data), $\mathrm{n}=101$ (final data), Data expressed in $\%$ and analysed by Chi square test, $\mathrm{x}^{2}$ 44.40, df- $1, \mathrm{p}<0.0001$

Fig No.6 and fig. No.7 depicted that in final data group duration of $\mathrm{O} 2$ used is $<2$ days ( $<1-2$ days) in significantly more number of cases $(69.70 \%)$ compared with that of basal data group which was taken prior to intervention. In basal data group duration of $\mathrm{O} 2$ used $>2$ days ( $>2-7$ days) in more percentage $(77 \%)$ of cases.

Fig. No.8 Outcome

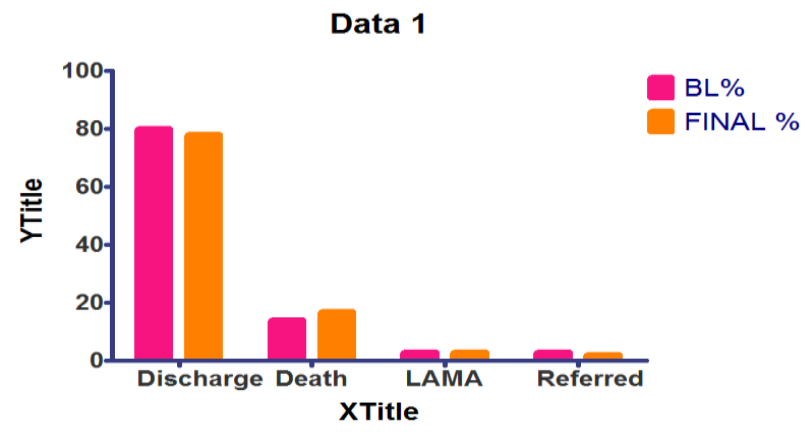

$\mathrm{N}=69$ (base line data), $\mathrm{n}=101$ (final data), Data expressed in $\%$ and analysed by Chi square test, $\mathrm{x}^{2}$ 0.5156 , df $-3, p>0.05$ 
In the above fig no.8, it was observed that there was no significant difference between the baseline data and final data at any type of outcome parameters like discharged, death, LAMA and referred.

\section{Discussion}

This study was conducted to implement a guide line for rational use of oxygen in sick neonates and optimise its use which reduces the use of resources as well as dangerous outcome of hyperoxia. The study result of base line data showed significant irrationality in $\mathrm{O} 2$ utilization in sick neonates which was not as per the WHO prescribed guide line ${ }^{[6,11]}$. The knowledge level of care givers like doctors and nurses was not updated regarding adverse consequences of excessive or suboptimal use of $\mathrm{O} 2$ therapy. Some times to satisfy the parents also they keep the patient under $\mathrm{O} 2$ therapy when there was no need. Oxidative stress is a serious outcome of hyperoxia due to excessive use of $\mathrm{O} 2$ during neonatal period and responsible for generalized tissue damage and in particular causes injury to lung, CNS, retina, red blood cells. Supplemental oxygen in new born care should be prudent to avoid changes and fluctuations in $\mathrm{SpO}_{2}{ }^{[3]}$.

In base line data more number of cases received $\mathrm{O} 2$ supplementation compared to that of final data which showed unawareness of health care givers in this facility and showed wastage of resources unnecessarily. Oxygen use indicators like emergency signs, specific signs, with minimum investigations were also not as per the guide line in base line data which was changed significantly in final data. Duration of therapy $>1$ day to 2 days observed in final data which was significantly lesser than that of baseline data i.e ranges from 3-5days. There is no check list of oxygen supply in base line data where as in final data there was significantly reduction of amount of $\mathrm{O} 2$ supply $^{[12],[15]}$.

Titration of $\mathrm{O} 2$ supply was made in final data by nasal prong at the rate of 0.5 litre/minute then supply seized without making any harm as per $\mathrm{O} 2$ supply guide lines.

Finally from study there was significant amount of reduction of $\mathrm{O} 2$ consumption compared with that of baseline data. This result is similar to other Indian study $^{[7]}$.

When at the end outcome in terms of death, discharge and LAMA were compared and showed no statistical significant difference between the base line and final data. This indicates that increasing the duration and concentration of $\mathrm{O} 2$ supply could not improve outcome parameters rather lead to some complications ${ }^{[5],[13],[14],[16],[17] .}$

An important obstacle was faced by the team members during the implementation of this QI strategy that some of the staff members were not willing rigidly to change. This was tackled by motivating them, explaining them the importance of rational use of $\mathrm{O} 2$ by showing different study results.

\section{Limitations of the Study}

The limitations of the study were shot duration study, no long term follow up of the patient for any late consequences of irrational use of $\mathrm{O} 2$. The specific organ level tissue injuries were not evaluated.

\section{Conclusion}

Oxygen saturation monitoring should occur continuously in all newborns who have respiratory distress, or commence oxygen therapy.

The lowest oxygen saturation level recommended commencing oxygen therapy.

Understanding the effects of $\mathrm{O} 2$ administration is important for the management of oxygen therapy in preterm newborn in order to prevent inadvertent cellular and tissue damage caused by hyperoxemia, in the patients requiring supplemental oxygenation This small interventional study helped us in significantly improving the quality care regarding $\mathrm{o} 2$ use and prevents $\mathrm{O} 2$ related toxicity with available resources and manpower in resource poor setting.

\section{Suggestions}

$\mathrm{O} 2$ therapy guideline is must in every newborn units which can prevent toxicities related to over use of $\mathrm{O} 2$.Periodic orientation programme/CME should be conducted among teaching faculties, SR and PG 
students. Emphasis should be given for rational use of $\mathrm{O} 2$ during the $\mathrm{UG}$ and $\mathrm{PG}$ teaching programme. Judicious uses of oxygen are very important among preterm neonate for prevention of retinopathy of prematurity.

\section{References}

1. http://www.everypreemie.org/wpcontent/uploads/2017/07/Oxygen_7.6.17.pdf last assessed on 25.10.18

2. Melinda Paul, CRNP, NNP-BC. Oxygen Administration to Preterm Neonates in the Delivery Room: Minimizing Oxidative Stress .Advances in Neonatal Care. April 2015( 15 ) $2: 94-103$.

3. Perrone S, Bracciali C, Di Virgilio N, Buonocore G. Oxygen Use in Neonatal Care: A Two-edged Sword. Front. Pediatr. 2017;4:143. doi: 10.3389/fped.2016.00143

4. https://extranet.who.int/rhl/topics/newbornhealth/care-newborn-infant/whorecommendation-oxygen-therapy-pretermnewborns.Updated on $17^{\text {th }}$ Nov ,2015. Last assessed on 19.07.2019.

5. Yesmin Ozsureci and Kubra Aykac. Oxidative related diseases in newborn. Medicine and cellular longivity. 2016; article ID 2768365; 9 pages

6. Serafina Perrone, Maria Luisa Tataranno, Gemma Stazzoni, Giuseppe Buonocore. Oxidative stress and free radicles related diseases in newborn. Advances in Bioscience and Biotechnology. 2012; (3):1043-1050.

7. Sindhu Sivanandan, Tavpritesh sethi, Rakesh Lodha, Anu thukral, M Jeeva sankar, Ramesh Agarwal1, Vinod K Paul1 and Ashok K Deorari. Target Oxygen Saturation Among Preterm Neonates on Supplemental Oxygen Therapy: A Quality Improvement Study. Indian Paediatrics.2018; (55)

8. Lisa Baba, Jacqueline McGrath. Oxygen Free Radicals: Effects in the Newborn Period. Advance neonatal care. Nov.2008; 8(5): P. 256-264
9. Nicola Austin. Practice recommendation for Oxygen saturation targets for newborns cared for in neonatal units, on behalf of newborn clinical network clinical reference group; New Zealand, Review date 31.08.2017.

10. Improving the quality care for mother and newborn in health facilities: pocqi learners manual vol.2 2017

11. https://medlineplus.gov/ency/article/007242. htm,last updated 11.112018, last assessed on 19.07.2019

12. Augusto Sola. Oxygen Saturation in the Newborn and the Importance of Avoiding Hyperoxia-Induced Damage. Neo Reviews. July, 2015;(16)7. https://neoreviews.aappublications.org/conte nt/16/7/e406. last updated July,2015, Last assessed on 19.07.2019.

13. Bhandari V. Molecular mechanisms of hyperoxia-induced acute lung injury. Front. Biosci. 2008;13: 6653-6661.

14. O. F. Araneda, M. Tuesta.Lung Oxidative Damage by Hypoxia Oxidative Medicine and Cellular Longevity. Volume 2012, Article ID 856918, 18 pages

15. Oxygen therapy in newborn Neonatal clinical practice guidelines: Winnipeg regional health authority: appropriate date march 2015; page 1-4

16. Askie LM, Henderson-smart DJ, Irwing L, Simpson JN. Oxygen saturation targets and out comes in extremely preterm infants. New Engl. J Med 2003; 349: 959-967.

17. A.M. Pisochi and A. Pop. The role of antioxidants in the chemistry of oxidative stress: a review. European Journal of Medicinal Chemistry. 2015; 5( 97): 55-74. 